(C) 2021, The Authors. Published by Elsevier Inc. and Fass Inc. on behalf of the American Dairy Science Association ${ }^{\circledR}$. This is an open access article under the CC BY-NC-ND license (http://creativecommons.org/licenses/by-nc-nd/4.0/).

\title{
Investigating the cow skin and teat canal microbiomes of the bovine udder using different sampling and sequencing approaches
}

\author{
C. J. Dean, ${ }^{1} \oplus$ I. B. Slizovskiy, ${ }^{1}$ K. K. Crone, ${ }^{2} \oplus$ A. X. Pfennig, ${ }^{3} \odot$ B. J. Heins, ${ }^{4}{ }^{\oplus}$ L. S. Caixeta, ${ }^{1}{ }^{\oplus}$ \\ and N. R. Noyes ${ }^{1 *}$ (1) \\ ${ }^{1}$ Department of Veterinary Population Medicine, University of Minnesota, St. Paul, 55108 \\ ${ }^{2}$ Department of Biochemistry, Molecular Biology, and Biophysics, University of Minnesota, St. Paul, 55108 \\ ${ }^{3}$ Department of Biology, Georgia Tech University, Atlanta 30332 \\ ${ }^{4}$ Department of Animal Sciences, University of Minnesota, St. Paul 55108
}

\section{ABSTRACT}

There is a need for standardized, efficient, and practical sampling methods to support large population-based studies of the internal and external epithelial microbiomes of the bovine udder. The primary objective of this study was to evaluate different sampling devices for the isolation of microbial DNA originating from the internal and external teat epithelium. Secondary objectives were to survey and compare the microbial diversity of external and teat canal epithelial microbiomes using amplicon and shotgun metagenomic sequencing approaches. To address these objectives, we enrolled a convenience sample of 24 Holstein dairy cows and collected samples from the external epithelium at the base of udder, the external teat barrel epithelium, the external teat apex epithelium, and the teat canal epithelium. Extracted DNA was quantified and subjected to PCR amplification of the V4 hypervariable region of the $16 \mathrm{~S}$ rRNA gene and sequenced on the Illumina MiSeq platform (Illumina Inc., San Diego, CA). A subset of samples was subjected to a shallow shotgun metagenomic assay on the Illumina HiSeq platform. For samples collected from the external teat epithelium, we found that gauze squares consistently yielded more DNA than swabs, and Simpson's reciprocal index of diversity was higher for gauze than for swabs. The teat canal epithelial samples exhibited significantly lower diversity than the external sampling locations, but there were no significant differences in diversity between teat apex, teat barrel, and base of the udder samples. There were, however, differences in the microbial distribution and abundances of specific bacteria across external epithelial surfaces. The proportion of shotgun sequence reads classified as Bos

Received January 29, 2020.

Accepted August 10, 2020.

*Corresponding author: nnoyes@umn.edu taurus was highly variable between sampling locations, ranging from $0.33 \%$ in teat apex samples to $99.91 \%$ in teat canal samples. These results indicate that gauze squares should be considered for studying the microbiome of the external epithelium of the bovine udder, particularly if DNA yield must be maximized. Further, the relative proportion of host to non-host DNA present in samples collected from the internal and external teat epithelium should be considered when designing studies that utilize shotgun metagenomic sequencing.

Key words: udder microbiome, high-throughput sequencing, sampling technique, organic dairy

\section{INTRODUCTION}

High-throughput sequencing technologies have enabled the exploration of complex microbial communities, allowing researchers to establish links between the microbiome and animal health. With the affordability and accessibility of these sequencing technologies being at an all-time high, we are witnessing a surge in the number of studies exploring livestock-associated microbiomes (McFall-Ngai et al., 2013). The cow udder microbiome is no exception, and numerous recent studies have been published describing the composition of milk and the teat canal and teat apex epithelia (Falentin et al., 2016; Derakhshani et al., 2018; Andrews et al., 2019). Despite this growing interest, there has been limited published research on the appropriate design and execution of microbiome studies related to the cow udder.

Validated and reproducible research methods are crucial for maintaining both internal and external validity of microbiome studies (Schloss, 2018). Different sample collection methods, sample storage conditions, DNA extraction kits, and sequencing strategies can all significantly affect microbiome results, leading to an inability to interpret results across studies (Henderson et al., 2013; Salter et al., 2014; Kong et al., 2017; Kim et al., 2017). In some cases, these technical artifacts 
outweigh biological factors in determining differences in microbiome composition between studies (Holman et al., 2017; Randall et al., 2019). Therefore, it is crucial to establish robust and reproducible protocols that can be easily adopted by the scientific community within a given research area. This has been a focus and goal of several microbiome standard-setting bodies, including the Earth and Human Microbiome Projects (Turnbaugh et al., 2007; Gilbert et al., 2014). In some ways, research fields that are just beginning to integrate microbiome approaches are at an advantage because they can benefit from a unified methodology from the outset. Dairy cow udder microbiome research is in this phase and could reap long-term benefits from validated and standardized microbiome protocols. Importantly, such protocols must be well suited to on-farm sampling, and ideally will include modifications that support studies with very high sample numbers. This latter consideration is especially important because experiences in the human microbiome literature suggest that interindividual and temporal intraindividual microbiome variability is extremely high, necessitating repeated sampling and large sample sizes (Lloréns-Rico and Raes, 2019).

Microbiome studies that target low biomass samples are beset by additional challenges (Minich et al., 2018; Eisenhofer et al., 2019). First, researchers must ensure that samples yield sufficient quantity and quality of microbial DNA, which can be difficult in some environments (Minich et al., 2018). Second, results from low biomass samples are more likely to be affected by contaminating DNA during sample collection and processing (de Goffau et al., 2019). In some cases, even the so-called kitome (i.e., contaminants in DNA extraction kits and reagents) can overwhelm sample DNA, leading to confounded results (Salter et al., 2014). Premoistened swabbing remains one of the most established sample collection methods for the acquisition of microbial cells inhabiting low microbial biomass surfaces such as the human skin (Kong et al., 2017), and this approach has been adopted in the study of the bovine udder external epithelial microbiome (Verdier-Metz et al., 2012; Frétin et al., 2018). However, compared with human skin, the dairy cow udder and the sample collection environment present unique challenges (especially within the milking parlor), which make collection and downstream processing of external epithelial udder samples difficult (Metzger et al., 2018). For example, human skin microbiome studies often involve swabbing relatively large surface areas, with substantial pressure and vigorous rubbing and swabbing (Aagaard et al., 2013). The anatomy of the bovine udder presents trade-offs between sample collection efficiency and potential biological relevance. For example, if the scientific question focuses on the role of the microbiome as a risk factor in mastitis, the teat canal may be an important focal area to sample, given its role in preventing retrograde transport of bacteria up into the gland; indeed, previous studies have studied the teat canal microbiome in this context (Andrews et al., 2019). However, the teat canal is a relatively difficult epithelial site to access and could increase the risk of mastitis in sampled cows. The external teat apex may be a secondary sampling target, given that the teat orifice represents the first point of entry for mastitis-causing pathogens; in addition, the external teat apex would be easier to sample than the teat canal. However, the teat apex is a relatively small area, and it is not known whether swabbing of this specific area yields sufficient amounts of DNA. Alternatively, swabbing the entire teat barrel would increase the available surface area, but may not play as direct a role in mastitis dynamics as the teat apex and may not reflect the microbial ecology that occurs directly at the teat apex. Furthermore, the teats themselves are difficult to scrub vigorously with a swab because of their lack of hard structure and the fact that they hang suspended in air; to attain enough pressure, the sampler must use their non-sampling hand or another object to apply counterpressure during swabbing, which increases the risk of contamination. Finally, the udder itself could be a source of contamination because dirt, debris, and even hair may fall from these locations onto the sampling device.

Even when samples yield high amounts of DNA, it can be critical to distinguish whether such DNA is microbial or "host." This is especially true when relying on non-amplicon-based methods to conduct microbiome research. Although sequencing of $16 \mathrm{~S}$ rRNA gene amplicons is more commonly reported in the microbiome literature, shotgun metagenomic sequencing presents an opportunity to evaluate not only bacterial and archaeal community composition, but also functional genes, mobile genetic elements, and other uni- and multicellular organisms. The challenge for the shotgun metagenomics approach, however, is that samples with large amounts of host DNA can represent a huge inefficiency in sequencing because most of the generated sequence data will come from the host genome. These host-related sequences also decrease the ability to detect microbial DNA, especially from low-abundance microbes (Pereira-Marques et al., 2019). Although little has been described regarding the relative amounts of Bos taurus DNA within metagenomic samples from the external udder epithelium, the literature on human skin studies suggests that these samples could contain relatively large amounts of host DNA (Marotz et al., 2018), although the proportion varies greatly depend- 
ing on body site (Oh et al., 2014). Although various host-depletion or target enrichment methods exist to attempt to address this issue (Marotz et al., 2018; Nelson et al., 2019; Shi et al., 2019), none have been reported to be tested on bovine skin or milk samples.

Given these considerations, there is a need to evaluate sample collection and processing methods, as well as host DNA levels, for studies of the external bovine udder epithelium. Therefore, the main objective of this study was to evaluate different sampling protocolsspecifically anatomical sampling location and sampling device - for the isolation of microbial DNA from the external teat epithelium and teat canal of the bovine udder. Secondary objectives were to survey the composition and diversity of the cow udder microbiome using a combination of amplicon and shotgun metagenomic sequencing approaches.

\section{MATERIALS AND METHODS}

\section{Study Design}

Twenty-four organic dairy cattle were enrolled and sampled at the University of Minnesota West Central Research and Outreach Center (Morris, MN) on a single day in July 2018. Sample size was estimated using the Hotelling-Lawley trace test as implemented in GLIMMPSE (Kreidler et al., 2013), assuming repeated measures within cow and specifying the difference in Shannon diversity between sampling devices (gauze vs. swab) as the primary outcome of interest. Shannon's diversity was estimated at 10 units for gauze and 8 units for swab, based on previous reports of Shannon's diversity for teat canal microbiome samples (Falentin et al., 2016). Large but homoscedastic standard deviation was estimated at 1.5 units of Shannon diversity. At $\alpha=$ 0.05 and $\beta=0.20$, it was estimated that 20 cows would be required. Most animals were late-lactation cows that were close to being dried off within a few months of the study date. The cows were 194 DIM (SD = 91), SCC was $309 \times 10^{3}$ cells $/ \mathrm{mL}(\mathrm{SD}=360)$, and milk yield was $10 \mathrm{~kg} / \mathrm{d}(\mathrm{SD}=4.1)$. A barrier teat dip (1\% iodine) was used as the post-dipping solution in this herd (I-Deal Teat Dip, Ecolab Co., Mississauga, ON, Canada). Sampling procedures were designed to be congruent with previous studies of the dairy cow teat canal epithelium and milk microbiomes (Bouchard et al., 2015; Falentin et al., 2016). Samples were collected after cows had been brought into the parlor for morning milking, but before any predipping, forestripping, or milking occurred. The farm did not use a cow wash or rinse protocol before milking. Before sample collection, the udder and teats were assessed visually; if a large amount of debris was present on the udder or teats, a sterile pad was used to gently remove the debris. Cows were also assessed for signs of clinical mastitis, and cows displaying such signs were not enrolled in the study. After this visual assessment, enrolled cows were sampled with the following protocol: first, a sterile swab (cat no. C15053-006; Cardinal Health, Dublin, $\mathrm{OH}$ ) was used to swab the entire surface of 2 teats (either front or hind); second, a single 5.1- $\times$ 5.1-cm gauze square (cat. no. 067522; Honeywell North, Charlotte, NC) was used to wipe the entire surface of the remaining 2 teats (either front or hind); next, the teats were cleaned thoroughly with paper towels saturated with $70 \%$ ethanol; and finally, either a dry $(\mathrm{n}=6)$ or moistened $(\mathrm{n}=6)$ cytobrush (cat. no. C0012; CooperSurgical Inc., Trumbull, CT) was inserted $\sim 5 \mathrm{~mm}$ into each teat canal and turned twice $\left(720^{\circ}\right)$, similar to Falentin et al. (2016) and Bouchard et al. (2015). The second group of 12 cows was sampled as follows: first, a single gauze square was used to wipe the base of the udder in between the 4 teats; next, a single gauze square was used to wipe the surface of the distal one-third of the 2 hind teats; and finally, a swab was used to swab the distal one-third of the 2 front teats. These combinations (Table 1) were selected to prioritize comparison of (1) location (teat apex vs. teat barrel vs. udder base vs. teat canal), (2) device (swab vs. gauze of teat barrel and teat apex), and (3) surface area (front vs. hind teat barrels).

Between each sample, personnel changed gloves using aseptic gloving technique (i.e., using only the inside surface of the wrist area). All gauze squares and swabs were labeled as "sterile" by the manufacturer and were individually wrapped in sterile packaging. Sampling devices were individually opened just before sample collection and moistened with molecular biology-grade $1 \times$ PBS by dipping into a single-use container. Immediately after collection, all samples were placed into 1.5$\mathrm{mL}$ tubes containing molecular biology-grade $1 \times$ PBS solution. Swabs and cytobrushes were placed directly into the tubes, and the swab and cytobrush stems were cut using scissors that were sprayed with $70 \%$ ethanol between each sample. Gauze squares were rolled by the sample collector and placed into the $1.5-\mathrm{mL}$ tube using metal forceps that were sprayed with $70 \%$ ethanol between each sample. All sample tubes were then immediately placed on ice until arrival at the University of Minnesota, at which point they were stored at $-80^{\circ} \mathrm{C}$ until sample processing.

All samples were collected by a single veterinarianresearcher trained in cattle handling to minimize differences in sampling technique while ensuring worker safety. All cattle handling procedures were approved before the initiation of the study by the University of Minnesota Institutional Animal Care and Use Committee (Protocol Number: 1807-36109A). 


\section{DNA Extraction}

All sample processing occurred in a class II biological safety cabinet and was performed by a single laboratory technician wearing gloves and laboratory coat. Before any work, the work surface was decontaminated using UV radiation and 70\% ethanol. Genomic DNA (gDNA) extraction was performed using the Qiagen MagAttract PowerMicrobiome DNA/RNA KF Kit (cat. no. 27600-4-KF; Qiagen, Hilden, Germany). The protocol was followed according to manufacturer's recommendations. PowerBead 96-well plates (Qiagen) containing $0.1-\mathrm{mm}$ glass beads were loaded with thawed sterile-cut ends of the cytrobrush, swab, or gauze before beginning the protocol, with the addition of 650 $\mu \mathrm{L}$ of warmed $\left(60^{\circ} \mathrm{C}\right)$ Solution MBL/RNase A (Qiagen) into each well. Molecular-grade sterile water was added to 2 randomly selected sample wells to serve as negative controls (extraction blanks). To aid in cell lysis by mechanical forces, the plate was sealed and placed on a Mini-Beadbeater-96 (115 V, BioSpec Products, Bartlesville, OK). Bead beating took place according to manufacturer's recommendations: 30 -s bead beating phase at $645 \times g$ for 4 rounds. A 30-s pause between each round was used to prevent overheating. Following further processing and isolation steps, the resulting supernatant was transferred for magnetic bead purification using a MagMAX Express96Deep Well Magnetic Particle Processor (Applied Biosystems, Foster City, CA) and ClearMag Beads (Qiagen). Briefly, after binding, the DNA bound to the beads was subjected to 3 wash steps with ClearMag Wash Solution (Qiagen; washes 1 and 2), 200-proof molecular biology-grade ethanol (wash 3), allowed to dry for $5 \mathrm{~min}$, and eluted into $100 \mu \mathrm{L}$ of nuclease-free water. Following extraction, gDNA was stored at $-20^{\circ} \mathrm{C}$ until library preparation. Quality control measurements for the quantity of isolated gDNA were taken using the Qubit 4 Fluorometer (Invitrogen, Carlsbad, CA) with the Qubit dsDNA HS Assay Kit (Invitrogen). Additionally, sample gDNA integrity was determined by electrophoresis using the Genomic ScreenTape and Genomic DNA Reagents with the 4200 TapeStation System (Agilent, Santa Clara, CA).

\section{Library Preparation for Amplicon and Shotgun Libraries}

For amplicon sequencing, extracted DNA was subjected to PCR amplification and sequencing of the V4 hypervariable region of the $16 \mathrm{~S}$ rRNA gene as previously described (Gohl et al., 2016). For shotgun metagenomic sequencing, extracted DNA from each sample was quantified using a fluorimetric PicoGreen assay. Purity of DNA was measured using a NanoDrop spectrophotometer (Thermo Fisher Scientific, Waltham, MA). Samples with greater than $0.2 \mathrm{ng} /$ $\mu \mathrm{L}$ of DNA and having absorbance ratios at $260 / 280$ $\mathrm{nm}$ and $260 / 230 \mathrm{~nm}$ of $\sim 2.0$ were selected for library preparation using Illumina's NexteraXT DNA Sample Preparation Kit, following the manufacturer's protocols (cat. no. FC-130-1096; Illumina Inc., San Diego, CA).

\section{Amplicon and Shotgun Sequencing}

The 16S rRNA amplicon libraries were subjected to paired-end sequencing $(2 \times 300 \mathrm{bp})$ on the Illumina MiSeq (Illumina Inc.) platform using the MiSeq Reagent Kit v3 chemistry. Shotgun metagenomic DNA

Table 1. Sampling design and number of observations, by location, device, and quarter ${ }^{1}$

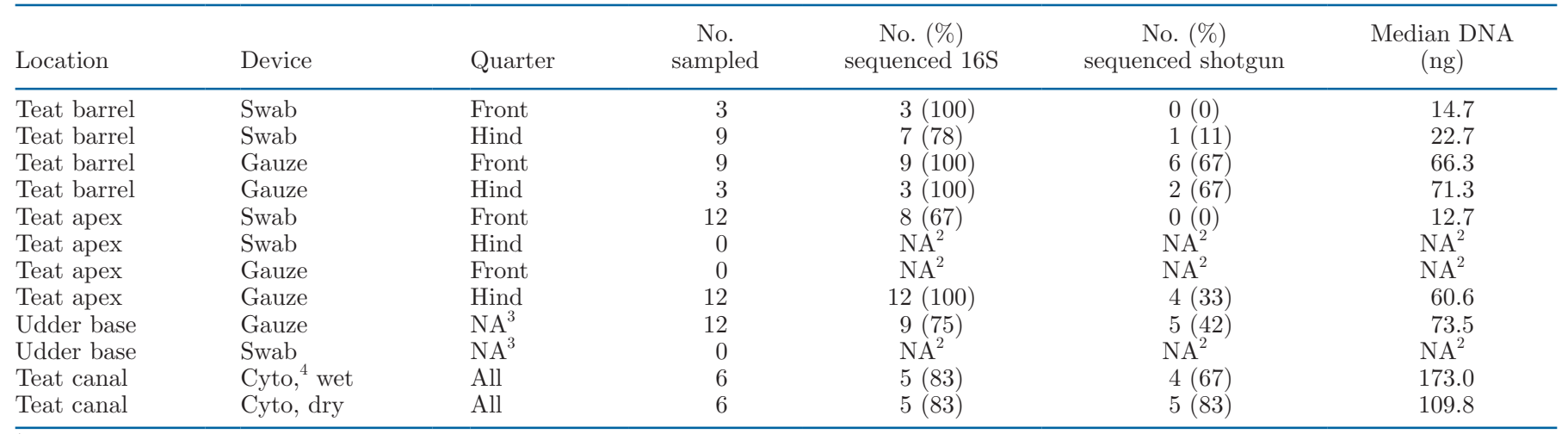

${ }^{1}$ The minimum requirements for $16 \mathrm{~S}$ rRNA and shotgun metagenomic sequencing were $0.2 \mathrm{ng} / \mu \mathrm{L}(\mathrm{DNA})$ and absorbance ratio at $260 / 280 \mathrm{~nm}$ and $260 / 230 \mathrm{~nm}$ of $\sim 2.0$

${ }^{2}$ Not assessed.

${ }^{3}$ Not applicable.

${ }^{4}$ Cytobrush. 
was subjected to paired-end sequencing $(2 \times 125 \mathrm{bp})$ on the Illumina HiSeq 2500 platform (Illumina Inc.) using the HiSeq SBS Kit v4 chemistry.

\section{Microbiome Data Analysis}

Paired-end sequence reads from each sample and both negative controls $(\mathrm{n}=63)$ were screened and processed using the DADA2 pipeline (Callahan et al., 2016). Primer sequences were removed from the forward and reverse read pairs using Cutadapt (Martin, 2011). Sequence reads were filtered for quality by visually inspecting the distribution of quality scores produced by DADA2. Forward reads were truncated to $240 \mathrm{bp}$ and reverse reads were truncated to $160 \mathrm{bp}$. Sequences with any ambiguous base pairs were removed. Forward and reverse reads with $>2$ expected sequencing errors were removed. Cleaned sequence reads were clustered and dereplicated into amplicon sequence variants (ASV). Dereplicated reads were denoised to remove and correct for sequencing errors. Denoised forward and reverse reads were aligned and combined into contigs using the default minimum overlap threshold of $12 \mathrm{bp}$. These contigs were then used to construct an ASV table; ASV $<250$ or $>256$ bp were removed from the table, as these were longer and shorter than the expected length of the sequenced amplicon. Chimeric sequences were identified and removed using the consensus method implemented in the "removeBimeraDenovo" procedure. The RDP Naive Bayesian Classifier algorithm was used to assign taxonomic units to ASV (Wang et al., 2007).

\section{Identification and Removal of Sequence Contaminants}

Putative sequence contaminants were identified using the "isContaminant" function implemented in "decontam" (Davis et al., 2018). Inputs to the function consisted of a count matrix of ASV abundances, $16 \mathrm{~S}$ qPCR concentrations (copies $/ \mu \mathrm{L}$ ) for each sample; and 2 negative control samples. We used $16 \mathrm{~S}$ qPCR concentrations to identify ASV whose relative abundances varied inversely with them (frequency-based method); and negative controls to identify ASV that had a higher prevalence in negative controls than in target samples (prevalence-based method; Davis et al., 2018). Score statistics generated from each method were then composited into a single score statistic and used for contaminant identification. A histogram of composite scores assigned to each ASV was generated and used as a guide to inform the appropriate classification threshold cutoff, as recommended by the software developers (Davis et al., 2018).

\section{Statistical Analyses}

For teat barrel and teat apex samples, comparison of DNA yield from gauze versus swabs was performed using a paired Wilcoxon signed-rank test. Because the surface area of the front versus hind barrels is known to differ (Zwertvaegher et al., 2012), the effect of quarter (front vs. hind) on DNA yield from the teat barrels was also compared using multivariable mixed models, with device and quarter as fixed effects and cow ID as random effect, as implemented in the "Imer" and "anova" functions in lme4 (Bates et al., 2015). The DNA yield from wet versus dry cytobrushes was compared using a nonpaired Wilcoxon signed-rank test.

Statistical analysis of microbiome data was performed in R (version 3.6.1, https://www.r-project.org/). Species diversity was estimated from the decontaminated ASV table using the "estimate_richness" function in Phyloseq (McMurdie and Holmes, 2013), following aggregation of sequence counts to the phylum, class, order, family, and genus levels. Sample-level $\alpha$ diversity was measured using Simpson's reciprocal index of diversity at each of these taxonomic ranks, including at the ASV level. To evaluate differences in $\alpha$ diversity between external teat epithelial samples (i.e., teat barrel vs. apex), we performed random effects modeling using the "lmer" function in lme4 (Bates et al., 2015), with cow ID specified as the random effect, and location, device, and quarters specified as fixed effects. The best-fit model was identified using a stepwise approach in which each variable was tested by comparing the fit of the full model versus the model with each variable removed, using the "anova" function in lme4, with maximum likelihood estimation. If the resulting $P$-value was $<0.10$, the variable was included in the final model. If more than one variable resulted in a significantly better model fit, then an interaction term was tested in the same manner. Once the final model was identified, post hoc Tukey's multiple comparison of means was performed using the "glht" function in package "multcomp" (Hothorn et al., 2008), and 95\% confidence intervals for the difference in means were obtained using the "confint" function in lme4. To identify differences in $\alpha$ diversity between the teat apex, teat barrel, udder base, and teat canal, a linear mixed-effects model was developed with cow as random effect and location as fixed effect; due to complete collinearity between the sample-device combinations of udder base-gauze and teat canal-cytobrush, "device" was not included as a fixed effect. Last, a separate Wilcoxon rank-sum test was performed on teat canal samples collected with a dry or wet cytobrush to determine whether the median microbial diversity was significantly different 
between the 2 sampling techniques. Beta diversity was visually assessed between covariables (i.e., location and device) using nonmetric multidimensional scaling (NMDS) plots of Bray-Curtis distances, following normalization of the ASV table using the cumulative sum scaling (CSS) procedure implemented in metagenomeSeq (Paulson et al., 2013). Before normalization, ASV present in $<10$ samples were discarded, as were negative controls and a single sample with a low read count (sample 23GU; read count $<5,000$ ). We used a permutational ANOVA (PERMANOVA) to test for differences in the microbial distribution and abundances between covariables. This involved two 2-way PERMANOVA tests using location and device as main effects, and two 1-way PERMANOVA tests using location as a main effect. For all PERMANOVA results, $P$ $<0.05$ was considered significant.

For differential abundance testing, normalized ASV counts were aggregated to the genus taxonomic levels using the "aggTax" function in metagenomeSeq. To compare abundances between wet and dry cytobrush samples taken from the teat canal, a zero-inflated Gaussian model was used ("fitZig" function) with device type ("wet" vs. "dry") as the independent variable and normalized counts as the dependent variable. To compare abundances between external sampling locations ("udder base," "teat barrel" and "teat apex") and device ("gauze" vs. "swab"), a zero-inflated Gaussian mixed model was used specifying location or device as the independent variable, normalized counts as the dependent variable, and cow ID as a random effect to account for repeated measures on cows. Random effects were controlled using the "duplicateCorrelation" function in limma, as integrated into the "useMixedModels" parameter of "fitZig" in metagenomeSeq. Empirical Bayes estimates for pairwise comparisons of log-fold differences in abundance were calculated using the eBayes function in limma. $P$-values were adjusted for multiple comparisons using Benjamini and Hochberg's method to control the false discovery rate.

\section{Bioinformatics and Statistical Analysis for Shotgun Metagenomic Data}

Shotgun metagenomic sequence data were processed with Trimmomatic to remove technical sequences and low-quality reads (Bolger et al., 2014). Using a custom reference database built from the Bos taurus reference genome (assembly ARS-UCD1.2) using Kraken2 (Wood et al., 2019), trimmed sequence reads that matched the B. taurus database were classified as "host," whereas all others were considered "non-host." Then, GNU core utilities were used to count the total number of host and non-host reads. Non-host reads were com- pared with a separate Kraken2 database consisting of archaeal (567 sequences), bacterial (42,231 sequences), viral (11,968 sequences), human (639 sequences), and protozoal (11,167 sequences) RefSeq libraries (https:/ /www.ncbi.nlm.nih.gov/refseq), as well as the UniVec and UniVec_Core libraries (9,230 sequences). In addition, 913 genomes from rumen-associated microbes were downloaded and included in this database to increase classification rates (Stewart et al., 2018). Classified sequence reads from kingdom down to the species level were assigned to operational taxonomic units (OTU), which we define here as the Kraken ID linked to the National Center for Biotechnology Information (NCBI) taxonomy to which each read aligned. Reported relative abundances of OTU were based solely on the proportion of non-host sequence reads that could be classified, not the total number of sequence reads in the data set.

\section{Data Availability}

All sequence data have been uploaded to the Sequence Read Archive on NCBI, under BioProject accession PRJNA512046 (https://www.ncbi.nlm.nih.gov/ bioproject/PRJNA512046). Biosamples were created separately for both amplicon and shotgun libraries, utilizing the MIMARKS and MIMS reporting guidelines developed by the Genomic Standards Consortium (Yilmaz et al., 2011).

\section{RESULTS}

\section{DNA Quantity and Quality}

In total, we collected 72 samples, 61 of which could be submitted for $16 \mathrm{~S}$ rRNA library preparation (Table 1), along with the 2 negative controls. The proportion of samples that could be submitted for both amplicon and shotgun metagenomic sequencing varied with device and was based on minimum DNA input requirements from the sequencing center of $>0.2 \mathrm{ng} / \mu \mathrm{L}$ DNA concentration and absorbance ratios at 260/280 nm and $230 / 280 \mathrm{~nm}$ of $\sim 2.0$ (Table 1 ). All teat apex and barrel samples collected with gauze could be submitted, whereas 18 out of 24 samples collected with swabs could be submitted. Among the teat barrel samples specifically, the 2 samples that could not be sequenced were both collected from the hind teats using swabs (Table 1). Among the external teat samples, 12 collected with gauze and 1 collected with swabs were of sufficient DNA quantity and integrity to meet the requirements for shotgun metagenomic library preparation (Table 1). From udder base samples, 9 could be submitted for amplicon sequencing and 5 for shotgun sequencing. 
Among teat canal samples, 5 collected with a dry and 5 collected with a wet cytobrush could be submitted for amplicon sequencing, with 5 dry and 4 wet cytobrush samples also meeting the shotgun sequencing requirements.

We found that the distribution of DNA yields within sampling location was highly variable between collection devices, which likely contributed to the variability in the proportion of samples that could be submitted for sequencing (Figure 1). Of the 72 samples collected, 66 yielded enough DNA for quantification using the Qubit fluorometer. For teat apex and barrel samples, the median DNA yield for quantifiable gauze squares was significantly higher than that for swabs (60.6 vs. $12.7 \mathrm{ng}$ and 68.8 vs. $21.2 \mathrm{ng}$, respectively; paired Wilcoxon signed-rank $P<0.05)$. For teat barrel samples, the quarters sampled (i.e., front vs. hind, Table 1) did not significantly affect DNA yield (ANOVA $P>0.05$, median for front quarters: $54.1 \mathrm{ng}$; for hind quarters: $40.3 \mathrm{ng})$. Within teat canal samples, the DNA yield for dry and wet cytobrushes was similar (145.6 ng vs. $173.0 \mathrm{ng}$, respectively; Wilcoxon $P>0.05)$. The median
DNA yield for samples collected from the udder base was $73.5 \mathrm{ng}$.

\section{Amplicon Sequencing}

Amplicon sequencing of the V4 hypervariable region of the 16S rRNA gene in the 61 target samples (i.e., non-control samples) yielded $5.8 \mathrm{M}$ amplicon sequence reads (median $87 \mathrm{~K}$, reads per sample, range $4 \mathrm{~K}$ to 253K, Supplemental Table S1; https://doi.org/10.3168/ jds.2020-18277). Following removal of primer pairs and low-quality sequence regions, $4.9 \mathrm{M}$ (median $75 \mathrm{~K}$, range $3 \mathrm{~K}$ to $218 \mathrm{~K}$ ) high-quality amplicon sequence reads were retained (Supplemental Table S1). Sequencing of 2 negative controls (extraction blanks) yielded a total of $53 \mathrm{~K}$ sequence reads.

\section{Amplicon Sequencing Classification Statistics}

Classification of sequence reads into ASV yielded 13,099 ASV distributed across the 4 sampling locations: teat apex $(\mathrm{n}=7,548)$, teat barrel $(\mathrm{n}=8,525)$, teat

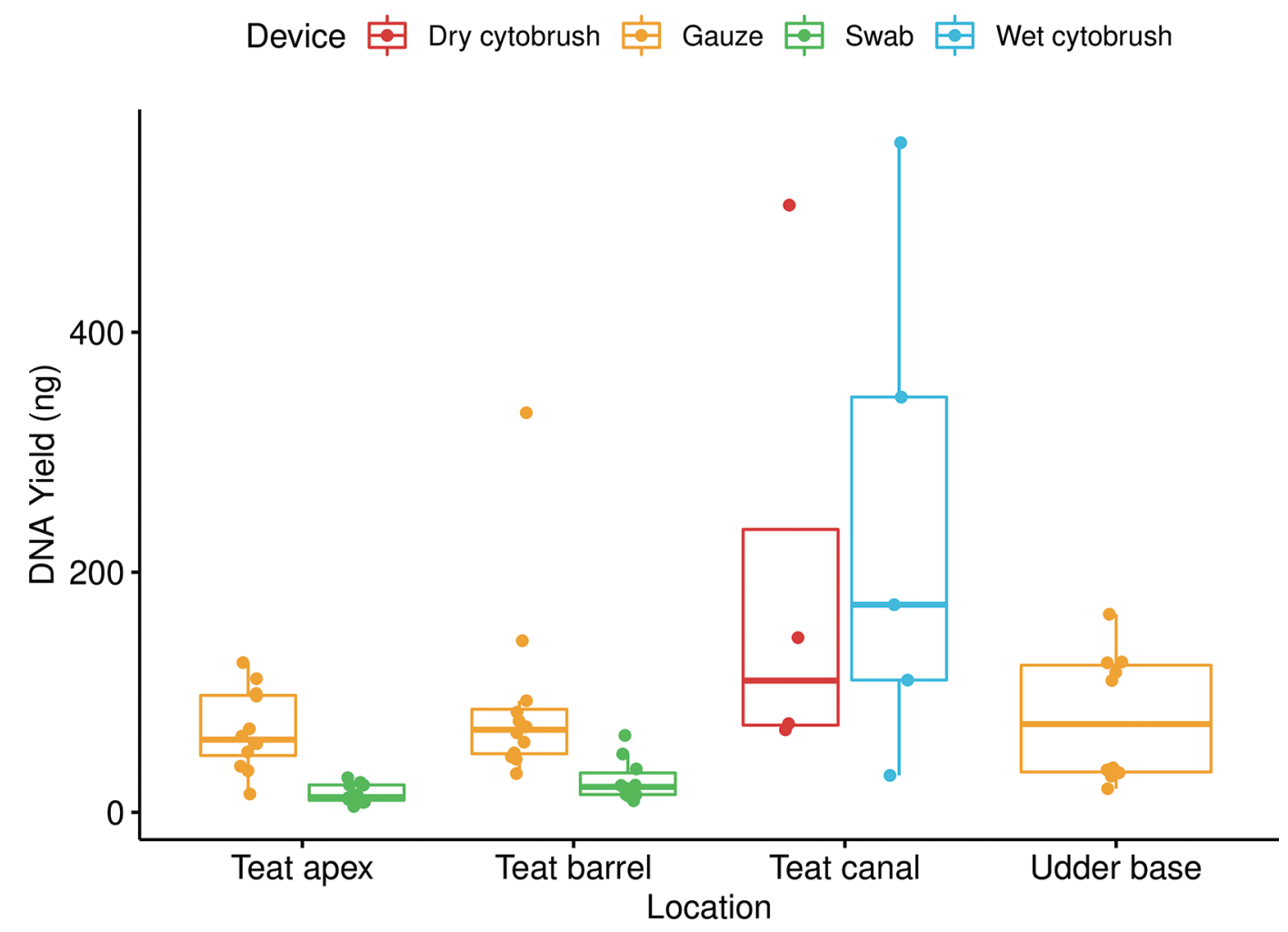

Figure 1. Box plots showing DNA yield (ng) as a function of sampling location. Points represent individual samples, boxes represent the interquartile range (IQR), whiskers represent the range of values extending beyond the lower (Q1 $-1.5 \times$ IQR) and upper quartiles $(\mathrm{Q} 3+1.5$ $\times$ IQR), and points extending beyond or below the whiskers represent outliers. Horizontal lines within each box represent median DNA yield values. Each box is colored according to the device used to collect the sample. For visualization purposes, we omitted one sample collected from the teat canal with a dry cytobrush due to its high DNA yield value of $36,200 \mathrm{ng}$, but it was included in all statistical analyses. 
canal ( $\mathrm{n}=2,841)$, and udder base $(\mathrm{n}=4,469)$. Assignment of ASV to different levels of taxonomy showed a consistent classification rate across each location, with the proportion of positive classifications (i.e., anything other than "unclassified") decreasing as a function of increased taxonomic resolution (Supplemental Table S2; https://doi.org/10.3168/jds.2020-18277).

\section{Taxonomic Composition and Analysis of Negative Controls}

Sequencing of the 2 negative controls (i.e., extraction blanks) yielded 588 ASV. The first negative control (NC1) consisted of $321 \mathrm{ASV}$ and the second negative control (NC2) consisted of 294 ASV. The number of ASV unique to NC1 and NC2 was 294 and 267, respectively, leaving 27 shared ASV between the 2 samples. The number of ASV shared with target samples was 165 for NC1 and 207 for NC2. The predominant phyla found in negative controls were Actinobacteria (NC1: 67.8\%, NC2: 26.2\%), Bacteroidetes (NC1: 14.3\%, NC2: 1.5\%), Firmicutes (NC1: 8.6\%, NC2: $60.2 \%$ ) and Proteobacteria (NC1: 5.4\%, NC2: 3.5\%) (Supplemental Figure S1; https://doi.org/10.3168/jds.2020-18277). The predominant genera found in negative controls were Corynebacterium (NC1: 13.4\%, NC2: 2.0\%), Streptomyces (NC1: 12.7\%, NC2: 2.4\%), Janibacter (NC1: 10.4\%, NC2: <1.0\%), Salinicoccus (NC1: 0.0\%, NC2: $7.8 \%$ ), Oceanobacillus (NC1: $0.0 \%, \mathrm{NC} 2: 7.5 \%$ ), and Clostridium_XI (NC1 <1.0\%, NC2: 4.4\%) (Supplemental Figure S2; https://doi.org/10.3168/jds.2020-18277).

\section{Contamination Identification and Removal}

The distribution of composite scores assigned to each ASV using "decontam" (Davis et al., 2018) showed a bimodal distribution, with 2 modes at 0.8 and 1.0 (Supplemental Figure S3; https://doi.org/10.3168/jds .2020-18277), indicating that most ASV were likely noncontaminants. A strict classification threshold of 0.6 was used to separate ASV into their respective components, with ASV below this threshold being discarded from further analysis. This resulted in the removal of 616 ASV from our data set, many of which were low abundance (median $6.06 \times 10^{-7} \%$, range $5.01 \times 10^{-7}$ to $0.002 \%$ ) and low prevalence (median 2 of 61 samples, range 2 to 38 of 61 samples).

\section{S-Based Microbial Richness and Diversity}

We explored microbial diversity across all taxonomic ranks using Simpson's reciprocal index of diversity (Figure 2). Among external teat samples (i.e., teat barrel and apex samples), the effect of quarter (i.e., front vs. hind) was not significantly associated with microbiome diversity at any taxonomic level and thus was not included in any final model (ANOVA $P>0.1$ ). At the ASV level among external teat samples, both device and sampling location were associated with diversity (ANOVA $P<0.1$ ), but their interaction was not (ANOVA $P>0.1$ ). Swab samples taken from the external teat exhibited lower inverse Simpson's diversity than gauze samples (Tukey's post hoc contrast -48.6 units, 95\% CI: -53.1 to $0.90, P=0.07$ ), and barrel samples exhibited significantly lower diversity than apex samples (Tukey's post hoc contrast: -26.1 units, $95 \%$ CI: -71.2 to -27.44$)$. Sampling location (i.e., teat apex vs. barrel) was only significantly associated with diversity at the ASV level, and not at the genus, family, order, class, or phylum levels. Device, however, remained significantly associated with external teat diversity at the genus and family levels (ANOVA $P<$ 0.05 ), but not at the order, class, and phylum levels; at both the genus and family levels, gauze yielded consistently higher diversity than swab samples.

Across all 61 samples, location was significantly associated with ASV-level $\alpha$ diversity (omnibus ANOVA $P<0.0001$ ), although these results were not adjusted for potential confounding by sampling device due to collinearity (i.e., the teat canal samples were collected with cytobrushes and the udder base samples were collected only with gauze). Specifically, the teat canal samples contained significantly lower ASV diversity than the udder base, teat barrel, and teat apex samples. Although the teat apex samples contained higher diversity than the udder base samples, which contained more than the teat barrel samples, these differences were not significant upon Tukey's post hoc testing.

\section{S-Based Taxonomic Composition}

To define the bacterial community structure, we classified ASV into taxonomic units at the kingdom, phylum, class, order, family, and genus levels. Across each sampling location, ASV with identical taxonomies were aggregated at each of these taxonomic ranks and filtered to only include those ranks (e.g., phyla, classes, orders, families, and genera) having an average relative abundance $\geq 1 \%$. The ASV not meeting this requirement were assigned a pseudo-taxonomic rank of Other. We visualized our results using 100\% stacked bar charts, sorted by the relative abundances of the taxa within each taxonomic rank.

We found that Actinobacteria, Firmicutes, and Proteobacteria were the predominant phyla across all locations (Figure 3). Firmicutes was the majority phylum, accounting for an average relative abundance greater than $50 \%$ across all sampling locations: teat 
apex $(54.3 \%)$, teat barrel $(50.6 \%)$, teat canal $(67.8 \%)$, and udder base (57.5\%). Among the dominant ASV found within Firmicutes, the genera Clostridium_XI, Romboutsia, and Turcibacter were consistently found across all sampling locations. Staphylococcus and Streptococcus were also highly prevalent but varied widely in their abundances across location, accounting for a mean abundance of 29.6 and $9.6 \%$, respectively, in teat canal samples, but less than 3 and $1 \%$, respectively, across the external epithelial samples. Actinobacteria was also highly abundant across locations, with most of these taxonomic units being assigned to the Corynebacterium genus. Proteobacteria was the next most abundant phylum, with Acinetobacter accounting for a mean relative abundance $>1 \%$ across all locations. Lower abundant ASV consisted of those assigned to the Candidatus Saccharibacteria and Chloroflexi phyla. These were highly prevalent across all sampling loca- tions, but varied slightly in their mean abundances, with Candidatus Saccharibacteria accounting for $1.6 \%$ of the taxonomic composition in teat apex and teat barrel samples and less than $1.0 \%$ in teat canal and udder base samples. Similar trends were observed for Chloroflexi, accounting for 1.5 and $2.2 \%$ of the composition in teat apex and teat barrel samples, respectively, but less than $1 \%$ in teat canal and udder base samples.

\section{Effects of Sampling Location and Device on 16S-Based Community Structure}

To visualize relationships between covariables (i.e., sampling location and device), we generated NMDS plots of Bray-Curtis distances from CSS normalized read counts (Figure 4). A global comparison between all samples revealed that sampling location accounted for the largest variation $\left(P<0.001, \mathrm{R}^{2}=0.19\right)$, fol-

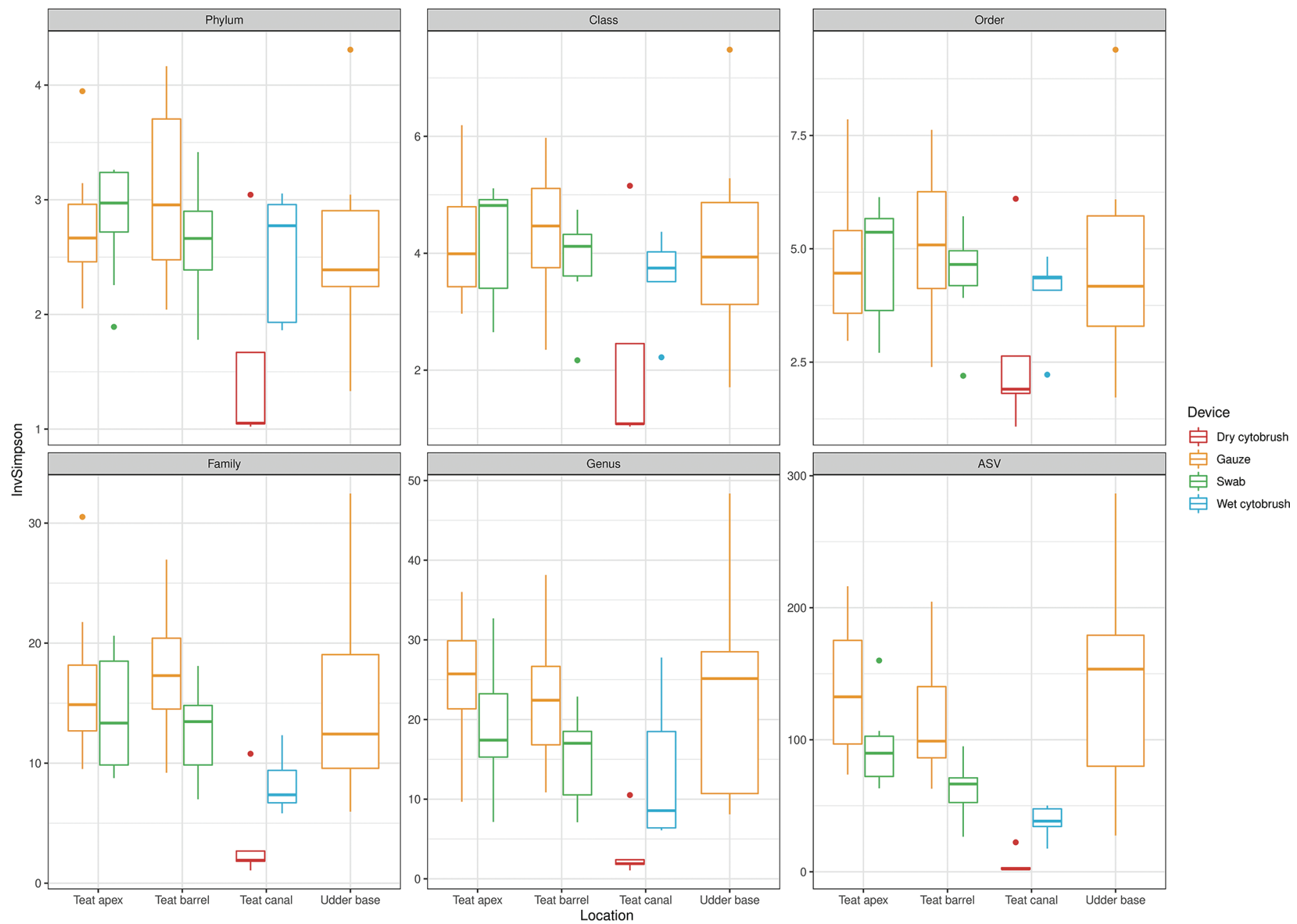

Figure 2. Box plots showing Simpson's Reciprocal Index of Diversity as a function of sampling location and taxonomic rank. Boxes represent the interquartile range $(\mathrm{IQR})$, whiskers represent the range of values extending beyond the lower $(\mathrm{Q} 1-1.5 \times \mathrm{IQR})$ and upper quartiles $(\mathrm{Q} 3+$ $1.5 \times \mathrm{IQR}$ ), and points extending above or below the whiskers represent outliers. Horizontal lines within each box represent median diversity values. Each box is colored according to the device used to collect the sample. 

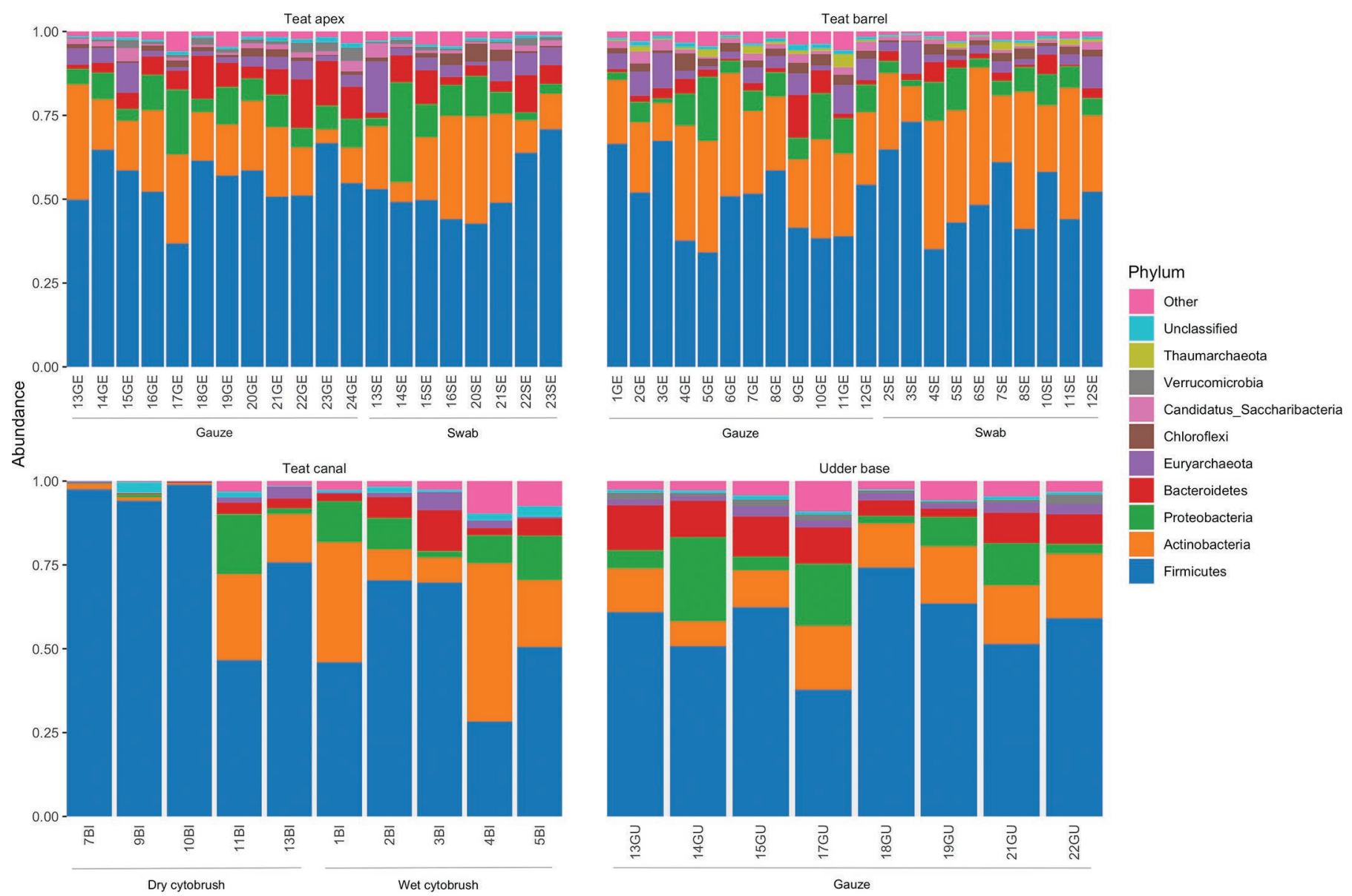

Figure 3. Relative abundance plots of amplicon sequence variants (ASV) aggregated at the phylum level for each location sampled. Classified phyla with an average relative abundance $\geq 1 \%$ within sampling locations are shown. Classified phyla with an average relative abundance $<1 \%$ were grouped into the "Other" category; ASV lacking a phylum-level classification were grouped into the "Unclassified" category. Labels along the $\mathrm{x}$-axis correspond to sample identifiers. Horizontal lines along the $\mathrm{x}$-axis are labeled with the device used to collect each sample. Negative control samples are not shown.

lowed by sampling device $\left(P<0.001, \mathrm{R}^{2}=0.10\right)$. Pairwise comparisons between samples collected from the teat apex and teat barrel revealed that location was a significant driver of the observed variation in the microbial community structure $\left(P=0.006, \mathrm{R}^{2}=\right.$ $0.07)$, as was sampling device $\left(P=0.018, \mathrm{R}^{2}=0.05\right)$. Within teat canal samples, there was a significant effect of sampling device $\left(P=0.009, \mathrm{R}^{2}=0.26\right)$. Microbial community structure also significantly differed between teat barrel and udder base samples $\left(P<0.001, \mathrm{R}^{2}\right.$ $=0.18)$, but not between teat apex and udder base samples $\left(P=0.46, \mathrm{R}^{2}=0.05\right)$.

\section{Differentially Abundant Taxa Across Sampling Locations and Devices, Based on 16S Data}

To determine the specific microbial players driving the observed differences in the microbial abundance and distribution between sampling locations and de- vices, we utilized a zero-inflated Gaussian model of the 16S count data (Paulson et al., 2013). We generated 5 models: 1 comparing abundances between wet and dry cytobrush samples collected from the teat canal, 3 comparing abundances between external sampling locations (teat apex, teat barrel, and udder base), and 1 comparing abundances from external teat epithelial samples collected with gauze versus swab. Abundance values were compared using log fold change ( $\mathbf{L o g} \mathbf{F C})$.

We identified 14 differentially abundant genera within teat canal samples (LogFC range: $2.88-8.23, P<0.05$ ). Five of these genera were significantly more abundant in samples collected with a dry versus wet cytobrush, with Staphylococcus (LogFC 8.23), Streptococcus (LogFC 5.36), and Pseudomonas (LogFC 3.55) likely driving many of the observed differences in the microbial distribution and abundances between devices. For external sampling locations, we identified 28 differentially abundant genera between udder base and teat apex samples 


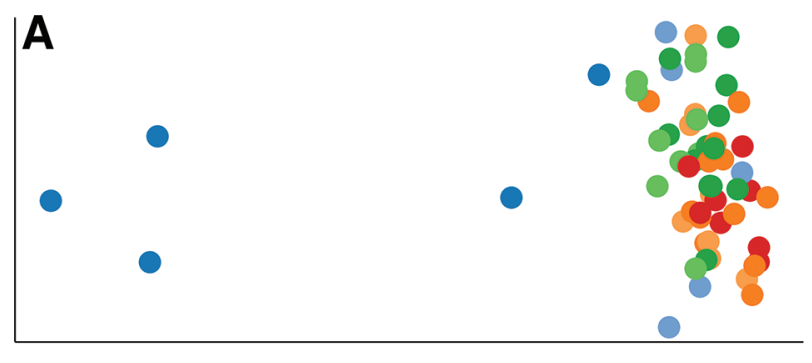

Device - Location
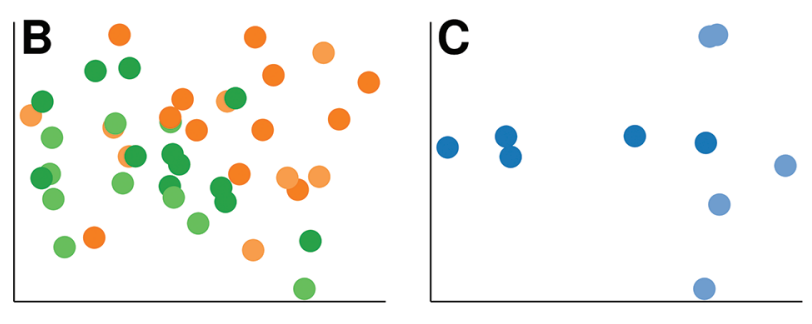

- Dry cytobrush - Teat canal

- Wet cytobrush - Teat canal

- Gauze - Teat apex

- Swab - Teat apex

- Gauze - Teat barrel

- Swab - Teat barrel
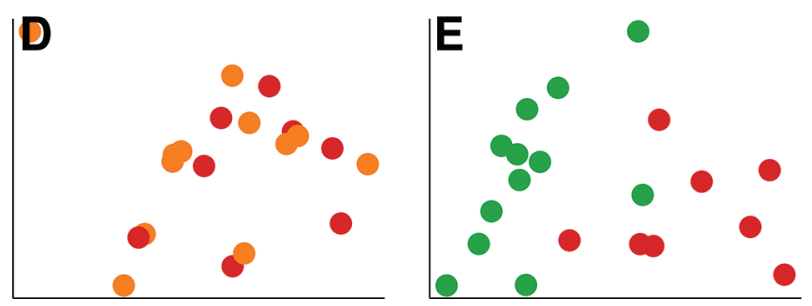

- Gauze - Udder base

Figure 4. Nonmetric multidimensional scaling (NMDS) plots of cumulative sum scaling normalized read counts of the cow udder microbiome. (A) NMDS plot of samples collected from all locations. Pairwise NMDS plots of (B) teat apex versus teat barrel (gauze and swab); (C) teat canal (dry versus wet cytobrush); (D) udder base versus teat apex (gauze); and (E) udder base versus teat barrel (gauze).

(LogFC range: 0.65-2.02), 103 between udder base and teat barrel samples (LogFC range: $0.60-2.64$ ), 61 between teat barrel and apex samples (LogFC range: $0.53-2.25$ ), and 58 between gauze and swabs ( $\log \mathrm{FC}$ range: $0.66-1.66)$. There were relatively few differences in microbial distribution and abundances of taxa originating from the udder base and teat apex, with many of the observed differences being due to low abundant taxa. Of the most abundant taxa identified in comparisons of the udder base and teat barrel, Turcibacter $(\operatorname{logFC} 1.19)$ and Nitrolancea $(\operatorname{LogFC} 1.56)$ were significantly more abundant in teat barrel samples, whereas Bacteroides ( $\log \mathrm{FC} 2.64)$, Alistipes ( $\log \mathrm{FC} 2.52)$, Papillibacter (LogFC 2.43), Oscillibacter (LogFC 2.40), and Akkermansia (LogFC 2.29) being more abundant in udder base samples. Corynebacterium ( $\log \mathrm{FC} 1.02)$, Nitrolancea (LogFC 1.15), and Janibacter (LogFC 1.10) were more abundant in teat barrel samples, whereas Bacteroides (LogFC 1.69), Akkermansia (LogFC 1.63), and Sporobacter (LogFC 1.18) were more abundant in teat apex samples. Among gauze and swab samples, Phascolarctobacterium (LogFC 1.17) were significantly more abundant in gauze and Turcibacter (LogFC 1.24) and Clostridium_sensu_stricto ( $\log \mathrm{FC} 1.05)$ were significantly more abundant in swab samples.

\section{Bovine Versus Microbial DNA from Shotgun Metagenomic Data}

We used a shallow shotgun metagenomic sequencing approach to define the distribution of bovine, microbial, and unclassifiable DNA originating from a subset of samples collected from the teat apex, teat barrel, teat canal, and udder base. Shotgun sequencing yielded $254 \mathrm{M}$ sequence reads across all 27 samples (median 9.5M reads per sample, range $6.6-11.5 \mathrm{M}$, Supplemental Table S2; https://doi.org/10.3168/jds.2020-18277). Following removal of adapters and low-quality sequence regions, 238M high-quality sequence reads were retained (median $8.8 \mathrm{M}$, range 6.1-10.9M, Supplemental Table S2). Trimmed sequence reads were then aligned to the Bos taurus reference genome and classified as bovine or non-host using Kraken2 (Wood et al., 2019). Using this approach, we found that the distribution of bovine versus non-host reads was highly variable across sampling locations (Figure 5), with the highest propor- 


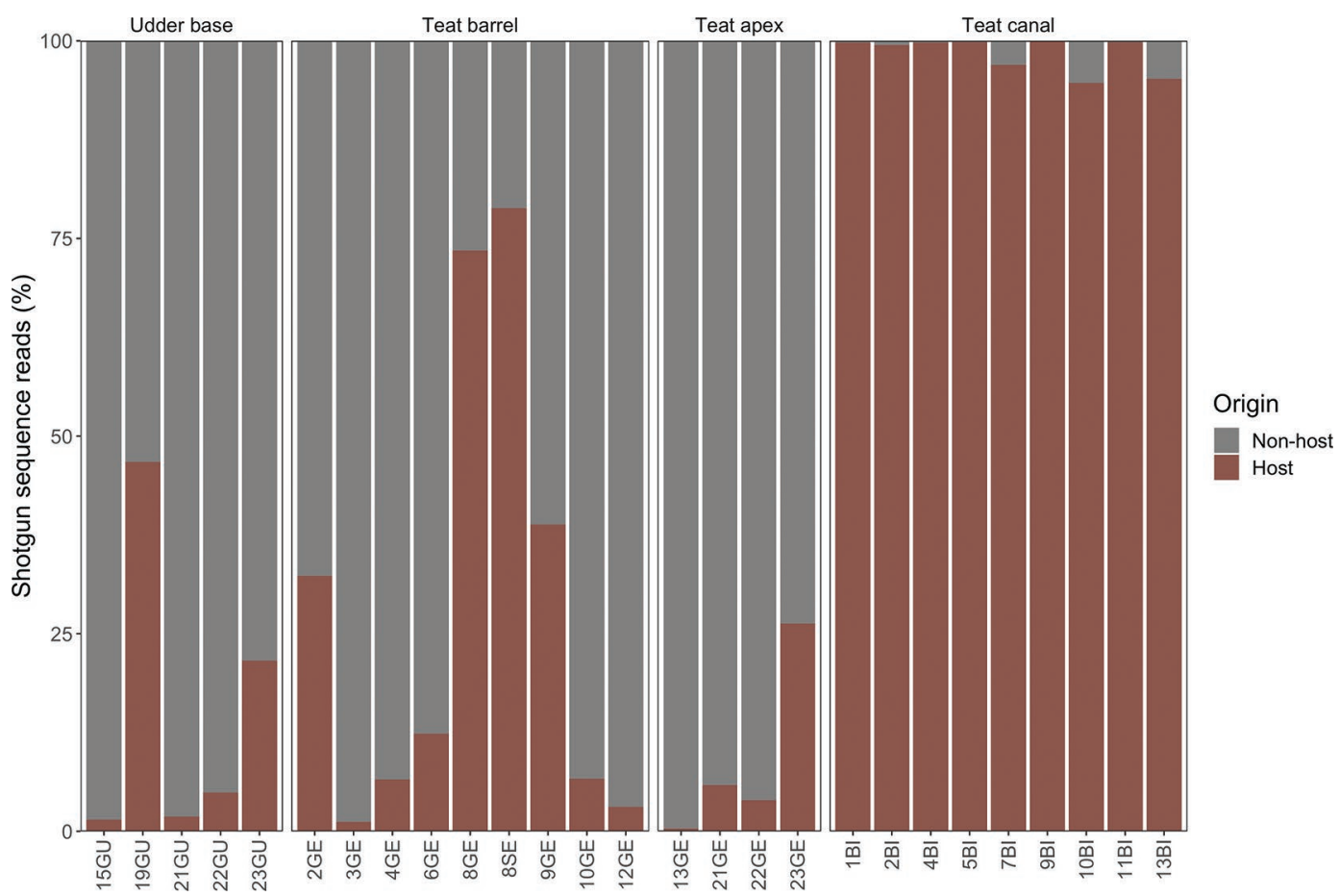

Figure 5. Stacked bar chart showing the percentage of shotgun sequence reads classified as bovine versus non-host as a function of the body site the samples were collected from. Shotgun sequence reads were trimmed and classified as host (brown) or non-host (gray) using Kraken2 (Wood et al., 2019). Labels along the x-axis correspond to sample identifiers.

tion of bovine reads being identified within the teat canal (median 99.79\%, range 94.66-99.91\%), followed by the teat barrel (median $12.34 \%$, range $1.18-78.82 \%$ ), udder base (median 4.91\%, range 1.48-46.71\%), and teat apex (median 4.90\%, range 0.33-26.27\%).

\section{Shotgun Metagenomic Taxonomic Composition}

Non-host reads (i.e., those not classified as Bos taurus) were aligned to a separate reference database consisting of archaeal, bacterial, human, protozoal, and viral genomes. The proportion of non-host reads that could be classified at any taxonomic level was variable across sampling locations (Supplemental Table S3; https:// doi.org/10.3168/jds.2020-18277), with the highest proportion of classified sequences coming from the teat barrel (median 21.2\%, range 12.2-31.1\%), followed by the teat apex (median $17.6 \%$, range $12.1-30.0 \%$ ) and udder base (median $14.5 \%$, range 11.9-24.7\%). Within the teat canal, this proportion varied by device, with dry cytobrush samples having a higher proportion of classified sequences (median $71.8 \%$, range $5.44-95.6 \%$ ) compared with samples collected with a wet cytobrush (median 13.8\%, range 9.15-20.8\%).

Non-host reads that could be classified into any taxonomic rank were clustered into 7,984 OTU. The taxonomic composition at the phylum level mirrored that of the $16 \mathrm{~S}$ data, with the majority of taxonomic units being assigned to Actinobacteria, Firmicutes, and Proteobacteria (Figure 6). However, the relative abundance of Actinobacteria was higher in shotgun data generated from the teat apex, teat barrel, and udder base samples, compared with that of the $16 \mathrm{~S}$ data, in which Firmicutes was the dominant phylum. The taxonomic composition at the genus/species level aligned with that found in the 16S data, with Acinetobacter, Corynebacterium, and Staphylococcus accounting for most of the classified sequence reads across locations. Using a shotgun metagenomics approach, we were able to acquire finer taxonomic resolution of these OTU (Table 2). Within teat apex and udder base samples, Acinetobacter lwoffii was ubiquitous and moderately abundant, accounting for a mean relative abundance of 1.7 and $1.6 \%$, respectively. Acinetobacter sp. SWBY1 was prevalent in teat apex samples, with a mean relative abundance of $1.0 \%$. Across all sampling locations, the most diverse OTU were assigned to the Corynebacterium genus, with Corynebacterium efficiens, C. marinum, C. maris, and $C$.xerosis constituting the majority (mean relative abundance $>1 \%$ ) of species-level identifications. Staphylococcus chromogenes, Staph. sciuri, and Streptococcus uberis were prevalent and highly abundant in teat canal samples, accounting for a mean relative abundance of $30.3,1.6$, and $3.8 \%$ of the sequence reads that could be 

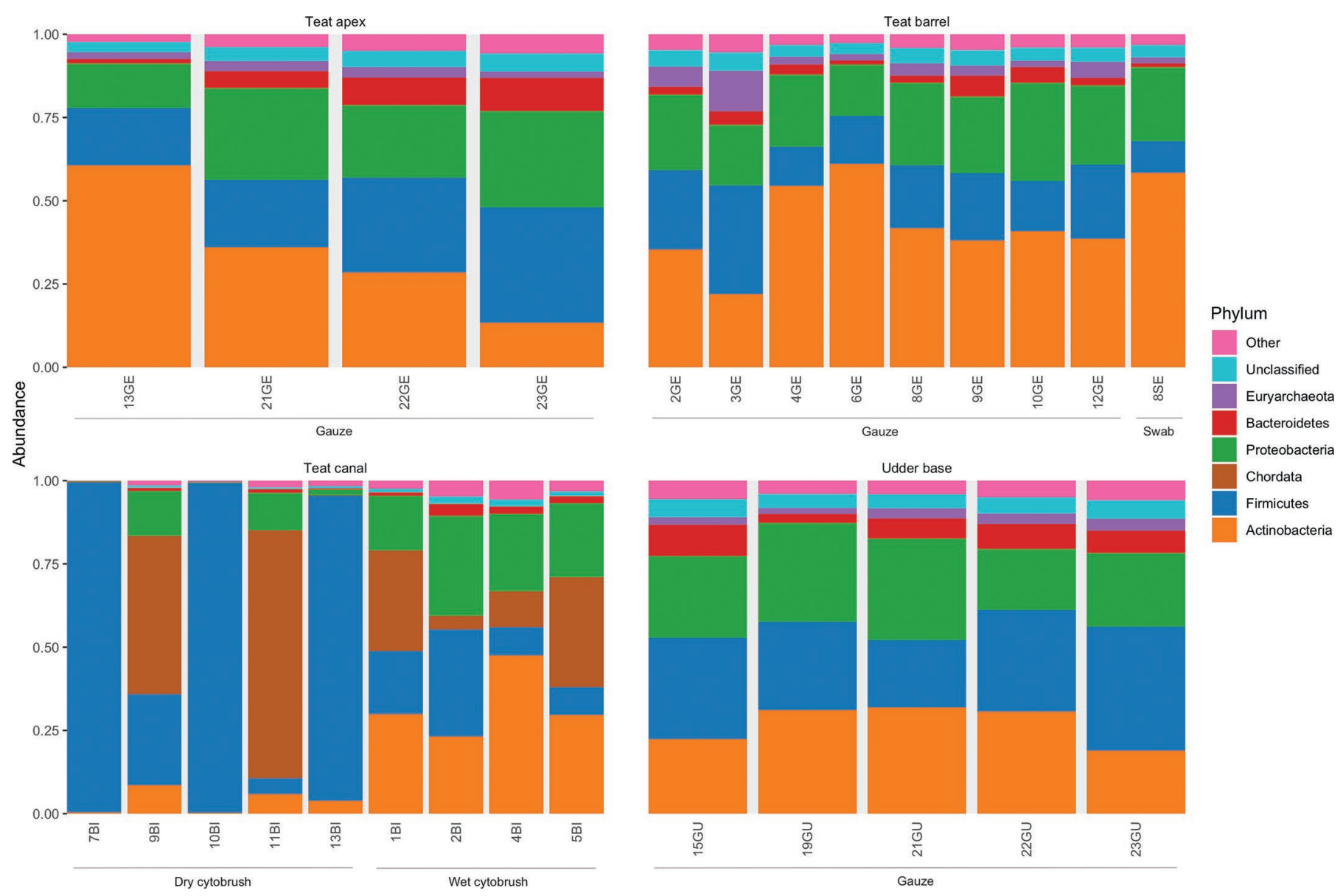

Figure 6. Relative abundance plots of shotgun sequence operational taxonomic units (OTU) aggregated at the phylum level for each location sampled. Classified phyla with an average relative abundance $\geq 1 \%$ are shown. Classified phyla with an average relative abundance $<1 \%$ were grouped into the "Other" category; OTUs lacking a phylum-level classification were grouped into the "Unclassified" category. Labels along the $\mathrm{x}$-axis correspond to sample identifiers. Horizontal lines along the $\mathrm{x}$-axis are labeled with the device used to collect each sample.

classified. Human DNA contamination was also evident throughout most samples but had a more dominant effect on samples collected from the teat canal (mean abundance $22 \%$, range $0.08-74.4 \%$ ) when the denominator included only classified sequence reads. However, when the denominator included all sequence reads, including Bos taurus and non-host, this proportion was lowered (median abundance $2.3 \times 10^{-5} \%$, range $1.9 \times$ $10^{-5}$ to $\left.2.9 \times 10^{-5} \%\right)$.

\section{DISCUSSION}

Sampling protocols are crucial for microbiome studies, and many questions arise when developing such protocols. Although the scientific literature addresses many of these considerations in relation to human skin microbiomes, the specifics of such protocols do not always translate well to on-farm sampling of bovids. To support large, population-based studies of bovine udder-associated microbiomes, we investigated the sampling and sequencing efficiency of combinations of sampling device and sampling location on the udder. We found that gauze squares consistently yielded more genomic DNA and higher microbial diversity than swabs, but that the differences in surface area between the front and hind quarters, as well as between the teat apex and teat barrel, did not affect DNA yield or observed microbial diversity. The lack of association between teats sampled (i.e., front vs. hind) and DNA quantity is somewhat unexpected given previous reports that the skin of the front teats yields significantly lower bacterial counts than that of the hind teats (Guarín et al., 2017). However, the lack of association in this study may have been a type II error given the relatively small sample size and unbalanced design. The significant effect of collection device on DNA quantity and microbial diversity may have been caused partially by differences in the collection device itself (e.g., larger 
surface area, differing amounts of exogenous DNA) and by differences in application of the device during sampling (i.e., different amounts of pressure applied during sampling, different surface area covered). We attempted to minimize systematic bias by collecting all samples in a uniform manner by a single researcher; by using individually packaged sterile swabs and gauze; and by utilizing best practices for microbiome research in terms of gloving and decontamination of instruments and work surfaces (Eisenhofer et al., 2019). However, some of the differences inherent in gauze versus swabs also present advantages for on-farm sampling. Specifically, we found that gauze squares support efficient and robust sampling of teats in the milking parlor, which may partially explain the observed differences in DNA yield and microbial diversity. First, the gauze material is more abrasive and easier to handle than swabs, which allowed us to apply more pressure and potentially more readily capture microbial cells tightly adhered to the teat. The ability to apply pressure on the skin during sample collection has been reported as an important factor in obtaining sufficient biomass from skin samples (Aagaard et al., 2013). Unlike human body sampling sites, the bovine teat is a pliable, pendulous structure suspended in air, which makes it difficult to apply vigorous pressure using a swab, unless the sampler rests the teat against a solid surface to apply counter-pressure while swabbing. However, this is not recommended due to the increased likelihood of contaminating the sampling site. In comparison, by using gauze squares, the sampler can effectively "grab" the teat between the gauze (using one hand) to apply firm pressure while wiping downward. This allows for increased pressure and a much simpler collection process. Second, the gauze material is less dense than a swab tip, which could allow for more efficient lysis of microbial cells during bead beating and thus detection of greater microbial diversity in the sample.

For sampling sites with a low microbial biomass, such as external and teat canal epithelium, it is critical that a sufficient amount of genomic material be recovered to meet the input and technical requirements of library preparation. Failure to meet this requirement results in samples that must be discarded from further downstream processing. This is especially true for shotgun metagenomics applications, which can accept a wide range of input DNA depending on the library preparation method used (e.g., enzymatic or mechanical shearing); however, there are tradeoffs between these approaches (Linnarsson, 2010; Head et al., 2014; Aigrain et al., 2016), and thus DNA extraction yield should be carefully optimized. Thus, while premoistened swabbing

Table 2. List of species that comprised an average relative abundance $\geq 1 \%$ within each sampling location

\begin{tabular}{lccc}
\hline \multicolumn{2}{l}{$\begin{array}{c}\text { Mean } \\
\text { Location and species }\end{array}$} & Range & Prevalence $^{2}$ \\
\hline Teat apex & & & \\
Acinetobacter lwoffii & 1.76 & $(0.32,3.57)$ & $4 / 4$ \\
Acinetobacter sp. SWBY1 & 1.09 & $(0.60,1.55)$ & $4 / 4$ \\
Corynebacterium efficiens & 3.99 & $(0.20,11.2)$ & $4 / 4$ \\
Corynebacterium marinum & 2.86 & $(0.057,5.67)$ & $4 / 4$ \\
Corynebacterium maris & 1.68 & $(0.40,4.62)$ & $4 / 4$ \\
Corynebacterium xerosis & 1.64 & $(0.26,2.36)$ & $4 / 4$ \\
Ornithinimicrobium flavum & 1.32 & & $4 / 4$ \\
Teat barrel & & $(0.32,6.31)$ & $9 / 9$ \\
Corynebacterium efficiens & 1.71 & $(1.14,10.7)$ & $9 / 9$ \\
Corynebacterium marinum & 4.76 & $(0.64,4.00)$ & $9 / 9$ \\
Ornithinimicrobium flavum & 2.30 & & \\
Teat canal & & $(0.002,3.04)$ & $9 / 9$ \\
Arcobacter trophiarum & 1.05 & $(0.0,7.15)$ & $9 / 9$ \\
Corynebacterium marinum & 1.22 & $(0.08,74.4)$ & $8 / 9$ \\
Corynebacterium xerosis & 1.39 & $(0.0,95.7)$ & $9 / 9$ \\
Homo sapiens & 22.2 & $(0.0,14.1)$ & $7 / 9$ \\
Staphylococcus chromogenes & 30.3 & & $6 / 9$ \\
Staphylococcus sciuri & 1.6 & $(0.39,3.16)$ & $6 / 9$ \\
Streptococcus uberis & 3.85 & $(0.18,4.31)$ & $5 / 5$ \\
Udder base & & $(0.25,4.92)$ & $5 / 5$ \\
Acinetobacter lwoffii & 1.60 & $(0.58,3.21)$ & $5 / 5$ \\
Aerococcus urinaeequi & 1.33 & $5 / 5$ \\
Corynebacterium efficiens & 1.61 & & $5 / 5$ \\
Corynebacterium marinum & 1.61 & & \\
Ornithinimicrobium flavum & 1.10 & & \\
\hline
\end{tabular}

${ }^{1}$ Mean abundance of all non-host classified reads.

${ }^{2}$ Prevalence refers to the number of samples in which each species was identified. 
remains one of the most established sample collection methods for the acquisition of microbial cells inhabiting low microbial biomass surfaces such as human and bovine skin (Verdier-Metz et al., 2012; Kong et al., 2017; Frétin et al., 2018; Andrews et al., 2019), gauze squares could be considered an alternative sample collection device. To support this recommendation, additional investigation of the relative contribution of sample versus exogenous DNA in gauze squares would be warranted, including a comprehensive analysis of negative controls. We also note that some of the meticulous sampling procedures used in this study may be incompatible with large-scale, rapid sampling within a commercial dairy. For example, we found that careful changing of gloves between each cow consumed a large portion of the percow time required for sampling. Although this practice is standard for microbiome studies, it may be valuable to compare human and intersample contamination rates when gloves are not changed between cows or, alternatively, if gloves are changed without using an aseptic gloving technique.

We showed that the microbes residing within and around the bovine udder and teats vary in their taxonomic composition and abundance between different anatomical locations of the bovine udder. Interestingly, microbial abundance and evenness (as measured by Simpson's reciprocal index of diversity) did not exhibit statistically significant differences between external sampling locations at any taxonomic level except ASV, but ordination analysis revealed that the structure of these communities was driven by distinct microbial players across anatomical locations. Consistent with previous findings, Firmicutes, Actinobacteria, Proteobacteria, and Bacteroidetes comprised the majority of phyla on the teat barrel, teat apex, and within the teat canal (Verdier-Metz et al., 2012; Falentin et al., 2016; Frétin et al., 2018). "Microbial dark matter" bacteria such as Candidatus Saccharibacteria were found in low abundance on the teat barrel (1.6\%), teat apex (1.6\%), and udder base samples (1.0\%; Marcy et al., 2007). It was also found within teat canal samples, albeit at a lower average relative abundance $(0.3 \%)$; however it is unclear whether this bacteria truly resides in the teat canal or whether it was inadvertently introduced by the cytobrush during sample collection (i.e., following contact with the teat skin).

Shotgun metagenomic sequencing revealed that the proportion of bovine versus microbial DNA was highly variable between sampling locations. This variability may have been caused by variable amounts of milk leakage that can occur, particularly when cows enter the milking parlor for routine milking. This was apparent during sampling, particularly for the teat canal epithelial samples, which often contained visible amounts of milk after sampling. This may explain why the teat canal samples consistently contained $>90 \%$ host DNA, which was significantly higher than the proportion of host DNA in external epithelial samples from the udder base and teats. Cytobrushes are also more abrasive than gauze squares and swabs, potentially causing more host epithelial cells to be scraped from the teat canal during sampling, leading to an increased proportion of host DNA in these samples. It may also be the case that the healthy teat canal is a low microbial biomass environment or even void of a functional microbiome, such that host DNA dominated these samples simply due to the low amounts of bacterial DNA in the teat canal itself. Finally, differences in the moisture content and type of epithelial cells lining the internal versus external teats may have contributed to the likelihood of capturing host cells during the sampling process, as has been demonstrated in human skin microbiome studies (Oh et al., 2014; Ross et al., 2017).

Compared with targeted sequencing of PCR amplicons, which only allow for taxonomic classification at a limited resolution, shotgun sequencing provides a unique opportunity to capture the entire genetic content (e.g., virulence factors, plasmids, antimicrobial resistance genes, and functional genes) of the underlying microbial community, making it possible to explore the functional potential of the community, while also acquiring finer taxonomic resolution of the sampled community. Using this tool, we showed that the taxonomic composition at the phylum level generally mirrored that of the $16 \mathrm{~S}$ data, a concordance that has been previously observed (Hillmann et al., 2018). However, there were some notable exceptions, with Firmicutes being the most abundant phylum in $16 \mathrm{~S}$ data and Actinobacteria the most abundant phylum in the shotgun metagenomic data. The reason for this lack of concordance may be the limited number of reference genomes available in NCBI's RefSeq, as evidenced by the large proportion of sequencing reads that were unclassified in our shotgun data set. Although this tool has not yet been fully utilized in the study of the cow udder microbiome, it could provide unique insights regarding the pathogens responsible for clinically important diseases affecting dairy cows, such as mastitis. For example, our finding that Staph. chromogenes dominated the classified microbial DNA in some teat canal epithelial samples warrants further investigation, although the presence of high proportions of bovine and unclassified DNA limits the interpretability of this finding. Similarly, our finding that human DNA constituted almost $25 \%$ of the classified sequence reads within the teat canal was surprising; however, bovine and human genomes do exhibit sequence similarity in some areas of their genomes (Everts-van der Wind et 
al., 2005; Zimin et al., 2009), so this observation may be partially explained by incomplete filtering of bovine DNA from our data set. Although a small proportion of human DNA was found in all shotgun samples, it likely had a more profound effect on abundance estimates of those samples collected from the teat canal simply because microbial diversity was lower and so few reads were classified as non-host. As has been discussed, the continued expansion of existing databases will support further metagenomic-based investigations (Doster et al., 2019). In addition, the shotgun metagenomic assay is relatively sensitive to the presence of large quantities of host DNA (compared with 16S rRNA), which could limit its use in locations such as the teat canal, a primary target for amplicon-based studies of the cow udder microbiome over the past several years (Gill et al., 2006; Bouchard et al., 2015; Falentin et al., 2016; Derakhshani et al., 2018). Although host-depletion assays do exist and have shown promising results in both the separation and degradation of host-cell nucleic acids originating from a variety of complex metagenomic samples (Marotz et al., 2018; Nelson et al., 2019), these have yet to be validated for samples originating from bovine epithelium. Therefore, careful study design considerations must be made before subjecting these types of samples to a shotgun metagenomic assay.

The effect of reagent contamination on the downstream analysis and interpretation of microbiome data sets originating from low microbial biomass samples has gained much attention over the last several years (de Goffau et al., 2019). Numerous papers have been published outlining best practices for both identifying and reporting library contaminants, such as including extraction blanks, sampling blanks, no-template amplification controls, and positive controls (Eisenhofer et al., 2019). New bioinformatic tools are also being developed to alleviate the putative effects that these technical artifacts can have in low microbial biomass environments (Davis et al., 2018). In our study, we reported on the taxonomic composition of negative control samples (extraction blanks) and found little evidence of significant reagent contamination in target samples following the application of "decontam" (Davis et al., 2018). Although we did not include sampling or no-template amplification controls, we did attempt to control for this by selecting a conservative classification threshold using "decontam" (Davis et al., 2018). However, future studies of the bovine udder epithelial microbiome - and particularly of the teat canal - would benefit from the inclusion of additional negative and positive controls to gauge background contamination introduced from the farm environment and chosen sample collection device, library reagent contamination, and sequencing efficiency.

\section{CONCLUSIONS}

In the present study, we aimed to evaluate the use of different sampling protocols and sequencing strategies to support future investigations into the cow udder microbiome. Our findings support the use of gauze squares as a practical and advantageous alternative to current sampling practices involving the use of swabs. Our findings also highlight the practical benefits and drawbacks of amplicon- and shotgun metagenomicbased sequencing approaches for studies of the cow udder microbiome, with some anatomical regions of the cow udder being more suitable for one approach over the other. Overall, we believe that our findings will aid in the future design and execution of microbiome studies targeting various regions of the cow udder.

\section{ACKNOWLEDGMENTS}

The authors thank the University of Minnesota Genomics Center and Minnesota Supercomputing Institute for assistance with library preparation, sequencing, and data storage. This research was funded by the Organic Agriculture Research and Extension Initiative (OREI) from the National Institute of Food and Agriculture (Washington, DC; grant no. 2018-51300-28563). The authors have not stated any conflicts of interest.

\section{REFERENCES}

Aagaard, K., J. Petrosino, W. Keitel, M. Watson, J. Katancik, N. Garcia, S. Patel, M. Cutting, T. Madden, H. Hamilton, E. Harris, D. Gevers, G. Simone, P. McInnes, and J. Versalovic. 2013. The Human Microbiome Project strategy for comprehensive sampling of the human microbiome and why it matters. FASEB J. 27:10121022. https://doi.org/10.1096/fj.12-220806.

Aigrain, L., Y. Gu, and M. A. Quail. 2016. Quantitation of next generation sequencing library preparation protocol efficiencies using droplet digital PCR assays - A systematic comparison of DNA library preparation kits for Illumina sequencing. BMC Genomics 17:458. https://doi.org/10.1186/s12864-016-2757-4.

Andrews, T., D. A. Neher, T. R. Weicht, and J. W. Barlow. 2019 Mammary microbiome of lactating organic dairy cows varies by time, tissue site, and infection status. PLoS One 14:e0225001. https://doi.org/10.1371/journal.pone.0225001.

Bates, D., M. Mächler, B. Bolker, and S. Walker. 2015. Fitting linear mixed-effects models using lme4. J. Stat. Softw. 67:1-48. https:// doi.org/10.18637/jss.v067.i01.

Bolger, A. M., M. Lohse, and B. Usadel. 2014. Trimmomatic: A flexible trimmer for Illumina sequence data. Bioinformatics 30:21142120. https://doi.org/10.1093/bioinformatics/btu170.

Bouchard, D. S., B. Seridan, T. Saraoui, L. Rault, P. Germon, C. Gonzalez-Moreno, F. M. E. Nader-Macias, D. Baud, P. François, V. Chuat, F. Chain, P. Langella, J. Nicoli, Y. Le Loir, and S. Even. 2015. Lactic acid bacteria isolated from bovine mammary microbiota: Potential allies against bovine mastitis. PLoS One 10:e0144831. https://doi.org/10.1371/journal.pone.0144831.

Callahan, B. J., P. J. McMurdie, M. J. Rosen, A. W. Han, A. J. A. Johnson, and S. P. Holmes. 2016. DADA2: High-resolution sample inference from Illumina amplicon data. Nat. Methods 13:581-583. https://doi.org/10.1038/nmeth.3869. 
Davis, N. M., D. M. Proctor, S. P. Holmes, D. A. Relman, and B. J. Callahan. 2018. Simple statistical identification and removal of contaminant sequences in marker-gene and metagenomics data. Microbiome 6:226. https://doi.org/10.1186/s40168-018-0605-2.

de Goffau, M. C., S. Lager, U. Sovio, F. Gaccioli, E. Cook, S. J. Peacock, J. Parkhill, D. S. Charnock-Jones, and G. C. S. Smith. 2019. Human placenta has no microbiome but can contain potential pathogens. Nature 572:329-334. https://doi.org/10.1038/ s41586-019-1451-5.

Everts-van der Wind, A. E., D. M. Larkin, C. A. Green, J. S. Elliott, C. A. Olmstead, R. Chiu, J. E. Schein, M. A. Marra, J. E. Womack, and H. A. Lewin. 2005. A high-resolution whole-genome cattle-human comparative map reveals details of mammalian chromosome evolution. Proc. Natl. Acad. Sci. USA 102:18526-18531. https:// doi.org/10.1073/pnas.0509285102.

Derakhshani, H., J. C. Plaizier, J. De Buck, H. W. Barkema, and E. Khafipour. 2018. Composition of the teat canal and intramammary microbiota of dairy cows subjected to antimicrobial dry cow therapy and internal teat sealant. J. Dairy Sci. 101:10191-10205. https://doi.org/10.3168/jds.2018-14858.

Doster, E., P. Rovira, N. R. Noyes, B. A. Burgess, X. Yang, M. D. Weinroth, L. Linke, R. Magnuson, C. Boucher, K. E. Belk, and P. S. Morley. 2019. A cautionary report for pathogen identification using shotgun metagenomics; a comparison to aerobic culture and polymerase chain reaction for Salmonella enterica identification. Front. Microbiol. 10:2499. https://doi.org/10.3389/fmicb.2019 .02499 .

Eisenhofer, R., J. J. Minich, C. Marotz, A. Cooper, R. Knight, and L. S. Weyrich. 2019. Contamination in low microbial biomass microbiome studies: Issues and recommendations. Trends Microbiol. 27:105-117. https://doi.org/10.1016/j.tim.2018.11.003.

Falentin, H., L. Rault, A. Nicolas, D. S. Bouchard, J. Lassalas, P. Lamberton, J.-M. Aubry, P.-G. Marnet, Y. Le Loir, and S. Even. 2016. Bovine teat microbiome analysis revealed reduced alpha diversity and significant changes in taxonomic profiles in quarters with a history of mastitis. Front. Microbiol. 7. https://doi.org/10.3389/ fmicb.2016.00480.

Frétin, M., B. Martin, E. Rifa, V.-M. Isabelle, D. Pomiès, A. Ferlay, M.-C. Montel, and C. Delbès. 2018. Bacterial community assembly from cow teat skin to ripened cheeses is influenced by grazing systems. Sci. Rep. 8:200. https://doi.org/10.1038/s41598-017 $-18447-y$.

Gilbert, J. A., J. K. Jansson, and R. Knight. 2014. The Earth Microbiome project: Successes and aspirations. BMC Biol. 12:69. https: //doi.org/10.1186/s12915-014-0069-1.

Gill, J. J., P. M. Sabour, J. Gong, H. Yu, K. E. Leslie, and M. W. Griffiths. 2006. Characterization of bacterial populations recovered from the teat canals of lactating dairy and beef cattle by $16 \mathrm{~S}$ rRNA gene sequence analysis. FEMS Microbiol. Ecol. 56:471-481. https://doi.org/10.1111/j.1574-6941.2006.00091.x.

Gohl, D. M., P. Vangay, J. Garbe, A. MacLean, A. Hauge, A. Becker, T. J. Gould, J. B. Clayton, T. J. Johnson, R. Hunter, D. Knights, and K. B. Beckman. 2016. Systematic improvement of amplicon marker gene methods for increased accuracy in microbiome studies. Nat. Biotechnol. 34:942-949. https://doi.org/10.1038/nbt .3601 .

Guarín, J. F., C. Baumberger, and P. L. Ruegg. 2017. Anatomical characteristics of teats and premilking bacterial counts of teat skin swabs of primiparous cows exposed to different types of bedding. J. Dairy Sci. 100:1436-1444. https://doi.org/10.3168/jds.2016-11514.

Head, S. R., H. K. Komori, S. A. LaMere, T. Whisenant, F. Van Nieuwerburgh, D. R. Salomon, and P. Ordoukhanian. 2014. Library construction for next-generation sequencing: Overviews and challenges. BioTechniques 56:61-64, 66, 68. https://doi.org/10.2144/ 000114133

Henderson, G., F. Cox, S. Kittelmann, V. H. Miri, M. Zethof, S. J. Noel, G. C. Waghorn, and P. H. Janssen. 2013. Effect of DNA extraction methods and sampling techniques on the apparent structure of cow and sheep rumen microbial communities. PLoS One 8:e74787. https://doi.org/10.1371/journal.pone.0074787.
Hillmann, B., G. A. Al-Ghalith, R. R. Shields-Cutler, Q. Zhu, D. M. Gohl, K. B. Beckman, R. Knight, and D. Knights. 2018. Evaluating the information content of shallow shotgun metagenomics. mSystems 3:e00069-18. https://doi.org/10.1128/mSystems.00069 -18 .

Holman, D. B., B. W. Brunelle, J. Trachsel, and H. K. Allen. 2017. Meta-analysis to define a core microbiota in the swine gut. mSystems 2:e00004-17. https://doi.org/10.1128/mSystems.00004-17.

Hothorn, T., F. Bretz, and P. Westfall. 2008. Simultaneous inference in general parametric models. Biom J. 50:346-363. https://doi.org/ 10.1002/bimj.200810425.

Kim, D., C. E. Hofstaedter, C. Zhao, L. Mattei, C. Tanes, E. Clarke, A. Lauder, S. Sherrill-Mix, C. Chehoud, J. Kelsen, M. Conrad, R. G. Collman, R. Baldassano, F. D. Bushman, and K. Bittinger. 2017. Optimizing methods and dodging pitfalls in microbiome research. Microbiome 5:52. https://doi.org/10.1186/s40168-017-0267 -5 .

Kong, H. H., B. Andersson, T. Clavel, J. E. Common, S. A. Jackson, N. D. Olson, J. A. Segre, and C. Traidl-Hoffmann. 2017. Performing skin microbiome research: A method to the madness. J. Invest. Dermatol. 137:561-568. https://doi.org/10.1016/j.jid.2016.10.033.

Kreidler, S. M., K. E. Muller, G. K. Grunwald, B. M. Ringham, Z. T. Coker-Dukowitz, U. R. Sakhadeo, A. E. Barón, and D. H. Glueck. 2013. GLIMMPSE: Online power computation for linear models with and without a baseline covariate. J. Stat. Softw. 54:i10. https: //doi.org/10.18637/jss.v054.i10.

Linnarsson, S. 2010. Recent advances in DNA sequencing methodsGeneral principles of sample preparation. Exp. Cell Res. 316:13391343. https://doi.org/10.1016/j.yexcr.2010.02.036.

Lloréns-Rico, V., and J. Raes. 2019. Tracking humans and microbes. Nature 569:632-633. https://doi.org/10.1038/d41586-019-01591-y.

Marcy, Y., C. Ouverney, E. M. Bik, T. Lösekann, N. Ivanova, H. G. Martin, E. Szeto, D. Platt, P. Hugenholtz, D. A. Relman, and S. R. Quake. 2007. Dissecting biological "dark matter" with singlecell genetic analysis of rare and uncultivated TM7 microbes from the human mouth. Proc. Natl. Acad. Sci. USA 104:11889-11894. https://doi.org/10.1073/pnas.0704662104.

Marotz, C. A., J. G. Sanders, C. Zuniga, L. S. Zaramela, R. Knight, and K. Zengler. 2018. Improving saliva shotgun metagenomics by chemical host DNA depletion. Microbiome 6:42. https://doi.org/ 10.1186/s40168-018-0426-3.

Martin, M. 2011. Cutadapt removes adapter sequences from highthroughput sequencing reads. EMBnet. J. 17:10-12. https://doi .org/10.14806/ej.17.1.200.

McFall-Ngai, M., M. G. Hadfield, T. C. G. Bosch, H. V. Carey, T. Domazet-Lošo, A. E. Douglas, N. Dubilier, G. Eberl, T. Fukami, S. F. Gilbert, U. Hentschel, N. King, S. Kjelleberg, A. H. Knoll, N. Kremer, S. K. Mazmanian, J. L. Metcalf, K. Nealson, N. E. Pierce, J. F. Rawls, A. Reid, E. G. Ruby, M. Rumpho, J. G. Sanders, D Tautz, and J. J. Wernegreen. 2013. Animals in a bacterial world, a new imperative for the life sciences. Proc. Natl. Acad. Sci. USA 110:3229-3236. https://doi.org/10.1073/pnas.1218525110.

McMurdie, P. J., and S. Holmes. 2013. phyloseq: An R package for reproducible interactive analysis and graphics of microbiome census data. PLoS One 8:e61217. https://doi.org/10.1371/journal.pone .0061217 .

Metzger, S. A., L. L. Hernandez, J. H. Skarlupka, T. M. Walker, G. Suen, and P. L. Ruegg. 2018. A cohort study of the milk microbiota of healthy and inflamed bovine mammary glands from dryoff through 150 days in milk. Front. Vet. Sci. 5:247. https://doi.org/ 10.3389/fvets.2018.00247.

Minich, J. J., Q. Zhu, S. Janssen, R. Hendrickson, A. Amir, R. Vetter, J. Hyde, M. M. Doty, K. Stillwell, J. Benardini, J. H. Kim, E. E. Allen, K. Venkateswaran, and R. Knight. 2018. KatharoSeq enables high-throughput microbiome analysis from low-biomass samples. mSystems 3:e00218-e17. https://doi.org/10.1128/mSystems .00218-17.

Nelson, M. T., C. E. Pope, R. L. Marsh, D. J. Wolter, E. J. Weiss, K. R. Hager, A. T. Vo, M. J. Brittnacher, M. C. Radey, H. S. Hayden, A. Eng, S. I. Miller, E. Borenstein, and L. R. Hoffman. 2019. Hu- 
man and extracellular DNA depletion for metagenomic analysis of complex clinical infection samples yields optimized viable microbiome profiles. Cell Rep. 26:2227-2240.e5. https://doi.org/10.1016/ j.celrep.2019.01.091.

Oh, J., A. L. Byrd, C. Deming, S. Conlan, H. H. Kong, and J. A. Segre. 2014. Biogeography and individuality shape function in the human skin metagenome. Nature 514:59-64. https://doi.org/10 $.1038 /$ nature13786.

Paulson, J. N., O. C. Stine, H. C. Bravo, and M. Pop. 2013. Differential abundance analysis for microbial marker-gene surveys. Nat. Methods 10:1200-1202. https://doi.org/10.1038/nmeth.2658.

Pereira-Marques, J., A. Hout, R. M. Ferreira, M. Weber, I. PintoRibeiro, L.-J. van Doorn, C. W. Knetsch, and C. Figueiredo. 2019. Impact of host DNA and sequencing depth on the taxonomic resolution of whole metagenome sequencing for microbiome analysis. Front. Microbiol. 10:1277. https://doi.org/10.3389/fmicb.2019 .01277 .

Randall, D. W., J. Kieswich, J. Swann, K. McCafferty, C. Thiemermann, M. Curtis, L. Hoyles, and M. M. Yaqoob. 2019. Batch effect exerts a bigger influence on the rat urinary metabolome and gut microbiota than uraemia: a cautionary tale. Microbiome 7:127. https://doi.org/10.1186/s40168-019-0738-y.

Ross, A. A., A. C. Doxey, and J. D. Neufeld. 2017. The skin microbiome of cohabiting couples. mSystems 2:e00043-17. https://doi .org/10.1128/mSystems.00043-17.

Salter, S. J., M. J. Cox, E. M. Turek, S. T. Calus, W. O. Cookson, M. F. Moffatt, P. Turner, J. Parkhill, N. J. Loman, and A. W. Walker. 2014. Reagent and laboratory contamination can critically impact sequence-based microbiome analyses. BMC Biol. 12:87. https:// doi.org/10.1186/s12915-014-0087-z.

Schloss, P. D. 2018. Identifying and overcoming threats to reproducibility, replicability, robustness, and generalizability in microbiome research. MBio 9:e00525-e18. https://doi.org/10.1128/mBio.00525 $-18$.

Shi, X., C. Shao, C. Luo, Y. Chu, J. Wang, Q. Meng, J. Yu, Z. Gao, and Y. Kang. 2019. Microfluidics-based enrichment and wholegenome amplification enable strain-level resolution for airway metagenomics. mSystems 4:e00198-e19. https://doi.org/10.1128/ mSystems.00198-19.

Stewart, R. D., M. D. Auffret, A. Warr, A. H. Wiser, M. O. Press, K. W. Langford, I. Liachko, T. J. Snelling, R. J. Dewhurst, A. W. Walker, R. Roehe, and M. Watson. 2018. Assembly of 913 microbial genomes from metagenomic sequencing of the cow rumen. Nat. Commun. 9:870. https://doi.org/10.1038/s41467-018-03317-6.

Turnbaugh, P. J., R. E. Ley, M. Hamady, C. M. Fraser-Liggett, R. Knight, and J. I. Gordon. 2007. The Human Microbiome Project. Nature 449:804-810. https://doi.org/10.1038/nature06244.

Verdier-Metz, I., G. Gagne, S. Bornes, F. Monsallier, P. Veisseire, C. Delbès-Paus, and M.-C. Montel. 2012. Cow teat skin, a potential source of diverse microbial populations for cheese production. Appl. Environ. Microbiol. 78:326-333. https://doi.org/10.1128/ AEM.06229-11.
Wang, Q., G. M. Garrity, J. M. Tiedje, and J. R. Cole. 2007. Naïve Bayesian classifier for rapid assignment of rRNA sequences into the new bacterial taxonomy. Appl. Environ. Microbiol. 73:52615267. https://doi.org/10.1128/AEM.00062-07.

Wood, D. E., J. Lu, and B. Langmead. 2019. Improved metagenomic analysis with Kraken 2. Genome Biol. 20:257. https://doi.org/10 .1186/s13059-019-1891-0.

Yilmaz, P., R. Kottmann, D. Field, R. Knight, J. R. Cole, L. AmaralZettler, J. A. Gilbert, I. Karsch-Mizrachi, A. Johnston, G. Cochrane, R. Vaughan, C. Hunter, J. Park, N. Morrison, P. RoccaSerra, P. Sterk, M. Arumugam, M. Bailey, L. Baumgartner, B. W. Birren, M. J. Blaser, V. Bonazzi, T. Booth, P. Bork, F. D. Bushman, P. L. Buttigieg, P. S. G. Chain, E. Charlson, E. K. Costello, H. Huot-Creasy, P. Dawyndt, T. DeSantis, N. Fierer, J. A. Fuhrman, R. E. Gallery, D. Gevers, R. A. Gibbs, I. S. Gil, A. Gonzalez, J. I. Gordon, R. Guralnick, W. Hankeln, S. Highlander, P. Hugenholtz, J. Jansson, A. L. Kau, S. T. Kelley, J. Kennedy, D. Knights, O. Koren, J. Kuczynski, N. Kyrpides, R. Larsen, C. L. Lauber, T. Legg, R. E. Ley, C. A. Lozupone, W. Ludwig, D. Lyons, E. Maguire, B. A. Methé, F. Meyer, B. Muegge, S. Nakielny, K. E. Nelson, D. Nemergut, J. D. Neufeld, L. K. Newbold, A. E. Oliver, N. R. Pace, G. Palanisamy, J. Peplies, J. Petrosino, L. Proctor, E. Pruesse, C. Quast, J. Raes, S. Ratnasingham, J. Ravel, D. A. Relman, S. Assunta-Sansone, P. D. Schloss, L. Schriml, R. Sinha, M. I. Smith, E. Sodergren, A. Spor, J. Stombaugh, J. M. Tiedje, D. V. Ward, G. M. Weinstock, D. Wendel, O. White, A. Whiteley, A. Wilke, J. R. Wortman, T. Yatsunenko, and F. O. Glöckner. 2011. Minimum information about a marker gene sequence (MIMARKS) and minimum information about any $(\mathrm{x})$ sequence (MIxS) specifications. Nat. Biotechnol. 29:415-420. https:/ /doi.org/10.1038/nbt.1823.

Zimin, A. V., A. L. Delcher, L. Florea, D. R. Kelley, M. C. Schatz, D. Puiu, F. Hanrahan, G. Pertea, C. P. Van Tassell, T. S. Sonstegard, G. Marçais, M. Roberts, P. Subramanian, J. A. Yorke, and S. L. Salzberg. 2009. A whole-genome assembly of the domestic cow, Bos taurus. Genome Biol. 10:R42. https://doi.org/10.1186/ gb-2009-10-4-r42.

Zwertvaegher, I., S. Van Weyenberg, S. Piepers, J. Baert, and S. De Vliegher. 2012. Variance components of teat dimensions in dairy cows and associated factors. J. Dairy Sci. 95:4978-4988. https:// doi.org/10.3168/jds.2011-5296.

\section{ORCIDS}

C. J. Dean (๑) https://orcid.org/0000-0003-1242-5375

K. K. Crone ㄴ https://orcid.org/0000-0001-9888-4710

A. X. Pfennig ๑ https://orcid.org/0000-0002-0114-1812

B. J. Heins ๑ https://orcid.org/0000-0003-2186-9082

L. S. Caixeta (๑) https://orcid.org/0000-0001-9577-4989

N. R. Noyes () https://orcid.org/0000-0001-6149-1008 